\title{
MIELOCINTILOGRAFIA COM RIHSA NO DIAGNÓSTICO DE PROCESSOS PATOLÓGICOS INTRA.RAQUEANOS
}

\author{
H. DietZ * \\ V. SeIXAS ** \\ E. ZEITLER $* * *$ \\ R. WOLF ***
}

O estudo cintilográfico do espaço subaracnóideo espinhal mediante injeção intratecal de isótopos radioativos - mielocintilografia - é um método semiótico relativamente nôvo e de grande importância. Bauer e Yuhl ${ }^{2}$, em 1953, foram os primeiros que, após experiências com animais, relataram a aplicação clínica da seroalbumina marcada com Iôdo ${ }^{131}$. Seguiram-se publicações de outros autores, como Krupin ${ }^{14}$, Liass ${ }^{15}$, Gabelowa e col. ${ }^{10}$ e Koroljuk ${ }^{13}$, que empregaram o Radônio ${ }^{222}$, enquanto Liass e Smagin ${ }^{17}$ e Liass ${ }^{16}$ defendiam o uso do Xenônio ${ }^{133}$. À exceção dêstes autores russos, trabalharam os demais com a RIHSA (Bauer e Yuh ${ }^{3}$; Perryman e col. 02; Bell e Hertsch ${ }^{4}$; Pecker e Javalet ${ }^{19}$; Feliciano Pinto ${ }^{21}$; Baggio e col. ${ }^{1}$; Fränkel e Sosonkin ${ }^{9}$; Wackenheim e col. ${ }^{22,23}$; Hübner e Brown ${ }^{12}$ ).

O uso da RIHSA teve a preferência devido a certas particularidades, como sejam a curta meia vida de mais ou menos 8 dias, a rápida eliminação e a pequena sobrecarga radioativa imposta à medula espinhal. Estas características permitem o uso corrente da RIHSA no diagnóstico neurológico.

\section{MATERIAL E METODO}

Usamos êste método desde 1964 (Dietz e col. ${ }^{5},{ }^{6},{ }^{7} ;$ Zeitler e col. ${ }^{24}$ ). Nosso material compreende 214 mielocintilografias feitas em 200 pacientes.

Mediante punção lombar ou suboccipital são injetados 60 a 100 microcuries de RIHSA, fornecida em frascos com 100 microcuries de atividade em $1 \mathrm{ml}$ de solução protêica a 1\%. Esta solução é introduzida imediatamente após a injeção intratecal de $50 \mathrm{mg}$ de prednisolona, usada para profilaxia de reações alérgicas e efeitos colaterais (náuseas, dores lombares e meningismo). Com a injeção suboccipital, não só de RIHSA como também da prednisolona, êsses sintomas ocorrem de vez em quando, desaparecendo tão logo a substância se difunde no líquido cefalorraqueano. Sintomas colaterais mais graves ou complicações sérias não foram observadas.

O registro da radioatividade bem como a inscrição em papel ou filme são feitos pelo aparelho Magnascaner III e $\mathrm{V}$ (Picker), com o qual é possivel inscrever, em uma única sessão, não só o estilocintilograma (branco e prêto ou colorido) como o fotocintilograma.

Trabalho realizado com material da Clinica Neurocirúrgica da Universidade de Mainz, Alemanha (Diretor: Prof. Dr. K. Schürmann) e do Instituto de Radiologia da Universidade de Mainz (Prof. Dr. L. Diethelm): * Livre Docente da Clinica Neurocirúrgica; ** Assistente da Clínica Neurocirúrgica; *** Assistentes do Instituto de Radiologia. 


\begin{tabular}{lccc}
\hline & $\begin{array}{c}\text { N.o de } \\
\text { casos }\end{array}$ & Local da injeção \\
\cline { 4 - 5 } & 44 & 38 & 16 \\
Processos expansivos & 12 & 13 & 1 \\
Aracnoidites & 9 & - & 9 \\
Avulsões de raizes cervicais & 8 & 6 & 2 \\
Malformações & 127 & 128 & 1 \\
Hérnias de núcleo pulposo & 200 & 185 & 29 \\
\hline Totais & & & \\
\hline
\end{tabular}

Tabela 1 - Mielocintilografias (214) agrupadas de acôrdo com os processos patológicos e com o local de injeção da substância radioativa (RIHSA).

Durante o exame o paciente é colocado em decúbito ventral. Sôbre a coluna vertebral movimenta-se o cintilador que capta as radiações emitidas pela substância radioativa. Se fôr necessária a localjzação exata de um processo em relação a uma apófise espinhosa ou a um corpo vertebral, pode-se fazer a inscrição sôbre uma radiografia da coluna do paciente, ou simplesmente, superpôr o cintilograma a esta radiografia.

O primeiro cintilograma é registrado dentro das duas primeiras horas após a introdução da substância radioativa. De acôrdo com o resultado obtido no primeiro registro ou caso o problema clínico o exija, pode ser feito outro traçado após 5 a 8 horas. De qualquer maneira é feito rotineiramente um terceiro, como contrôle, 24 horas após a injeção.

Não havendo obstrução à passagem do liquido cefalorraqueano a substância radioativa injetada difunde-se por todo o canai vertebral dentro de 5 a 8 horas.

\section{RESULTADOS}

Como se pode vêr na figura 1 , a atividade de 80 microcuries de RIHSA injetada entre $L_{2}$ e $L_{3}$ difundiu-se caudalmente até o fundo de saco dural e, cranialmente, até à região torácica inferior, durante a primeira hora; cinco horas após registrava-se atividade em todo o canal vertebral. A velocidade de difusão da RIHSA no sentido craniocaudal pode ser observada nessa mesma figura: duas horas após a injeção a atividade é registrada no têrço inferior da região torácica; após 6 horas verifica-se sua presença na porção superior da região lombar e, após 24 horas, está a radioatividade difundida por todo o canal. Nesta altura observa-se a superposição da radioatividade do canal espinhal com a da circulação geral. Em alguns casos ocorre rápida difusão da RIHSA de maneira que, após 90 minutos, pode ser visibilizado todo o canal raqueano. Entretanto, a difusão da radioatividade na maioria dêstes casos é ainda insuficiente e, em conseqüência disso, não é satisfatória a qualidade da mielocintilografia. Existindo obstáculo total ao trânsito liquórico observa-se, em primeiro lugar, a parada da difusão da substância radioativa à altura do obstáculo. 
Depois de algum tempo a radioatividade é captada do outro lado do processo obstrutivo de maneira que podem ser determinadas a extensão e localização dêsse processo.

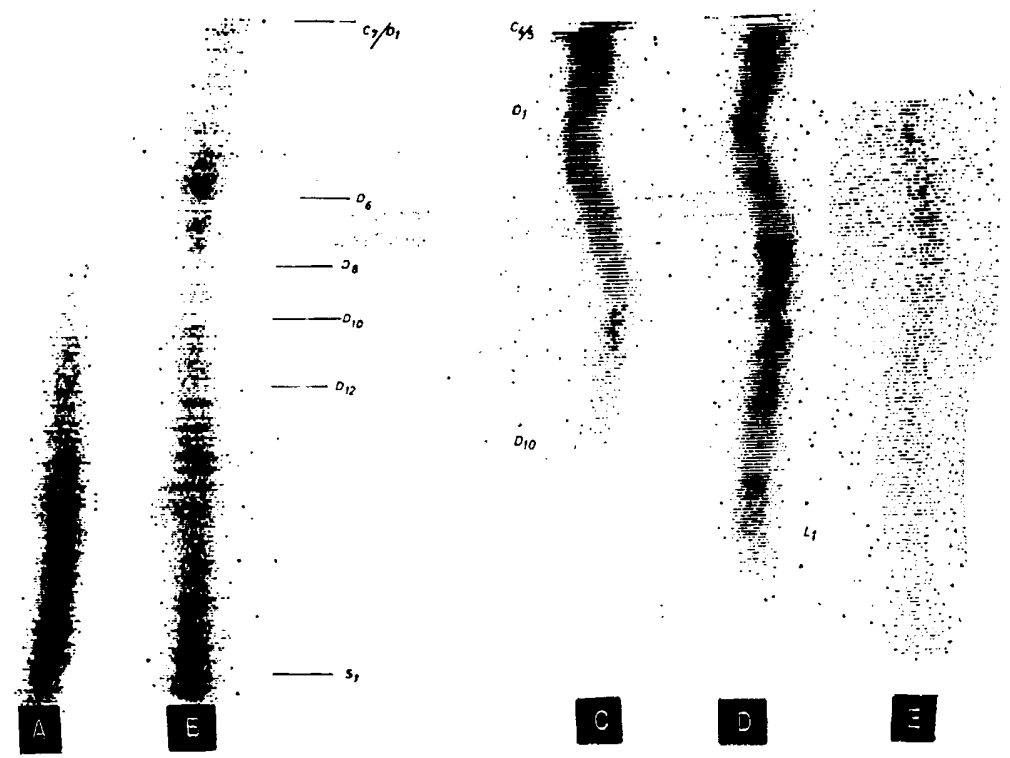

Fig. 1 - Em A, cintolograma mostrando a difusīo da RIHSA no sentido caudo-cranial uma hora após a injeção de 80 microcuries na altura de $L_{2} L_{3}$; em $B$, cintilograma do mesmo paciente 5 horas após a injeção; em $C, D$ e E, difusão da atividade de 100 microcuries de RIHSA no sentido crânio-caudal respectivamente 2,6 e 24 horas após a injecão suboccipital da substancia.

A figura 2 mostra três fotocintilogramas de uma mesma paciente, obtidas alguns minutos, 2 e 24 horas após a injeção suboccipital de 100 microcuries de RIHSA: com os dois primeiros foram determinados os limites superior $\left(\mathrm{C}_{7}\right)$ e inferior $\left(\mathrm{T}_{4}\right)$ de um tumor intramedular que produzia bloqueio total.

Nossa experiência permite concluir que a principal indicação para mielocintilografia é dada pelos processos que dificultam a circulação do líquido cefalorraqueano. Nestes casos é possivel, sem grande sobrecarga para o paciente, localizar um processo exp๕ınsivo, determinar sua extensão axial e verificar o grau de viabilidade do canal vertebral ao nível do bloqueio.

Entretanto é necessário analisar a mielocintilografia com espirito crítico, não the atribuindo propriedades e vantagens que ela na realidade não possui. Por exemplo, não podem ser identificados pequenos detalhes como os contornos de tumores, as formas de suas extremidades e os pequenos fundos de saco radiculares. 
No que concerne ao diagnóstico de localização de um processo expansivo, isto é, para saber se é intra ou extramedular, podemos afirmar que a mielocintilografia oferece ocasionalmente esta possibilidade. Esta afirmativa é demonstrada na figura 3A: duas horas após a injeção lombar de 90 microcuries de RIHSA o cintilograma mostrcu bloqueio total à altura de $\mathrm{T}_{10}$; o fotocintilograma mostrou, 24 horas depois, uma falha na faixa de atividade na mesma altura, do lado direito; 48 horas depois da injeção da substância radioativa, não houve difusão significativa desta acima de $\mathrm{T}_{9}-\mathrm{T}_{10}$. $\mathrm{O}$ ponto de referência para a determinação da altura da lesão foi tomado em relação aos processos espinhosos. A localização dêste processo estava plenamente de acôrdo $\mathrm{ccm}$ a sintomatologia: tratava-se de um neurinoma da 12 . $^{\mathrm{a}}$ raiz torácica direita, com os diâmetros aproximados de $4 \times 3 \times 2 \mathrm{~cm}$.

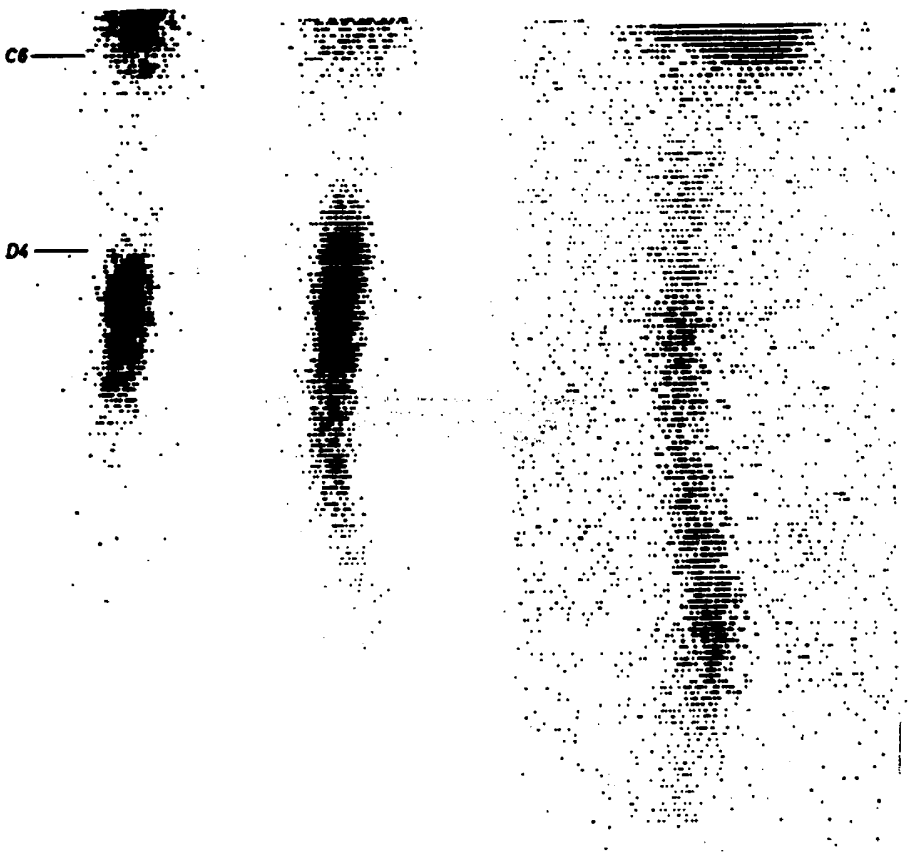

Fig. 2 - Cintilogramas registrados imediatamente, 2 e 24 horas após a injeção, por via suboccipital, de 100 microcuries de RIHSA em paciente de 54 anos com tumor intramedular $\left(C_{7}-T_{4}\right)$. A falha que se observa na coluna radioativa corresponde à extensão do tumor.

A figura $3 \mathrm{~B}$ apresenta o mielocintilograma em um caso de neurinoma da segunda raiz cervical direita, o qual se introduzia pelo forame occipital atingindo a cisterna magna. A radioatividade alcançou a altura do tumor 6 horas após a injeção lombar de 100 microcuries de RIHSA, difundindo-se 
a seguir e atingindo as cisternas basais. No local onde se encontrava o tumor havia uma falha na coluna radioativa correspondente ao tamanho do processo expansivo.

Da mesma maneira pode-se verificar a presença de processo localizado em vértebras e que diminua a luz do canal vertebral. Nestes casos a faixa de atividade apresenta-se com a forma de ampulheta, como se pode ver na figura $3 \mathrm{C}$, a qual mostra dois mielocintilogramas feitos, respectivamente, 4 e 24 horas após a injeção de 100 microcuries de RIHSA, à altura de $\mathrm{L}_{3}-\mathrm{L}_{4}$; tratava-se de uma estenose do canal raqueano devida a plasmocitoma do corpo da primeira vértebra lombar.
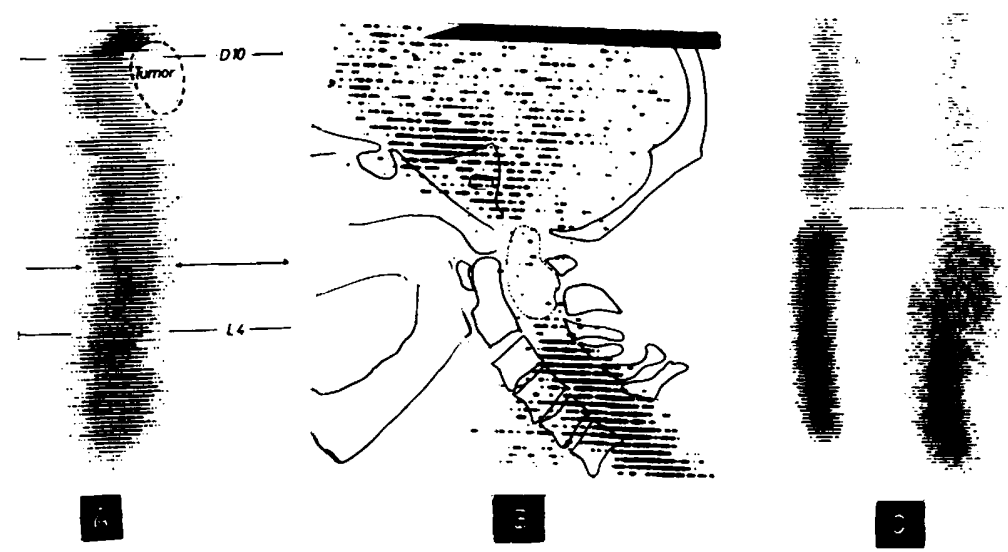

Fig. 3 - Em A, mielocintilograma mostrando falha na coluna radioativa na altura de $T_{10}$, demonstrando a existência de processo expansivo localizado nesta altura (neurinoma da 12.a raiz torácica). $E m$, mielocintilograma em um caso de neurinoma da 2.a raiz cervical direita que se introduzia pelo forame occipital, atingindo a cisterna magna. Em C, imagem em ampulheta mostrando a existência de processo localizado na 1.a vértebra lombar e obstruindo o canal raqueano (plasmocitoma); os mielocintilogramas foram registrados, respectivamente, $4 \mathrm{e}$ 24 horas após a injeção de RIHSA à altura de $L_{3}-L_{4}$.

No que tange aos processos inflamatórios da aracnóide que produzem obstrução ao trânsito do líquido cefalorraqueano, a mielocintilografia constitui recurso semiótico de importância, pois permite fazer o diagnóstico muito mais fácil e precocemente do que com os meios de contraste radiológicos. Além disso, é necessário considerar que restos do contraste que permaneçam no espaço subaracnóideo funcionarão como corpo estranho, aumentando o processo inflamatório. Em tais casos a mielocintilografia com RIHSA mostra não só o grau de alteração do trânsito liquórico, como também a extensão do processo. Achados cintilográficos positivos no diagnóstico de aracnoidites já foram descritos por Perryman e col. ${ }^{20}$, Feliciano Pinto ${ }^{21}$, Pantazis e col. ${ }^{18}$, Baggio e Morgando ${ }^{1}$ e Wackenheim ${ }^{22,}{ }^{23}$. Nossa experiência permite afirmar que podem ser observadas alterações caracte- 
rísticas no cintilograma em casos de aracnoidite (Fig. 4C): a largura e intensidade da faixa de radioatividade registrada serão tanto menores quanto mais intenso fôr o grau de obstáculo imposto à passagem liquórica pelo processo inflamatório. $O$ comprimento da coluna radioativa estreitada $\mathrm{e}$ de intensidade diminuida corresponde à extensão axial da aracnoidite (Dietz e col. ${ }^{7,8}$ ).

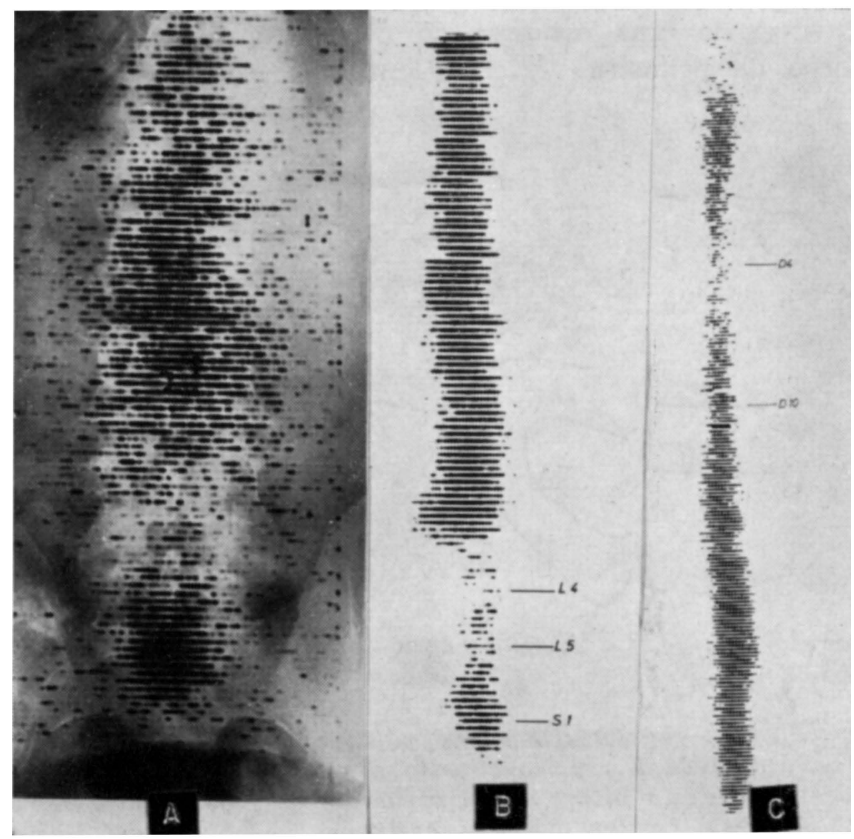

Fig. 4 - Em A, mielocintilograma superposto a uma radiografia simples da coluna vertebral; a falha de atividade que se observa na altura de $L_{4}-L_{5}$ corresponde a um prolapso discal dorsomedial; em $B$, mielocintilograma mostrando falha na coluna radioativa, na altura de $L_{4} e L_{5}$, correspondendo a um grande prolapso dorsolateral esquerdo; em $C$, diminuição da intensidade radioativa entre $T_{2}$ e $T_{10}$ correspondendo a uma aracnoidite pós-traumática.

Nos casos de malformações do canal vertebral (cistos dermóides, mieloceles e meningoceles) é importante fazer a mielocintilografia com o paciente em decúbito lateral para determinar a extensão e profundidade da lesão no plano sagital. O registro da atividade com o paciente nesta posição não é, entretanto, muito fácil devido à dificuldade em colocar o canal vertebral no plano de incidência da câmara de cintilação: com a movimentação do paciente poderão ser obtidos falsos registros da atividade, os quais serão tanto mais acentuados quanto maior fôr a distância entre o detetor e o canal raqueano. 
Especialmente bem demonstráveis pela mielocintilografia são as avulsões traumáticas de raízes cervicais. A representação cintilográfica da lesão assume a forma de uma extensão pontiaguda lateralmente à coluna radioativa. A figura 5A apresenta a imagem cintilográfica do fundo de saco correspondente à $7 .^{a}$ raiz cervical esquerda, obtida após a injeção suboccipital de 50 microcuries de RIHSA: o fundo de saco radicular se apresenta dilatado e vazio devido ao arrancamento da raiz correspondente. A cintilografia ajuda, nestes casos, a fazer o diagnóstico diferencial com a contusão do plexo braquial.

A maior parte de nossas mielocintilografias foi feita em casos de hérnia do núcleo pulposo da coluna lombar. Entretanto estas hérnias só são cintilogràficamente demonstráveis quando produzem redução acentuada da luz da canal vertebral à maneira de um processo expansivo.

As herniações pequenas, principalmente aquelas localizadas dorsolateteralmente, na maioria das vêzes passam despercebidas à mielocintilografia. Os prolapsos localizados na linha mediana, quando tenham certo volume, apresentam, à maneira de um tumor, um cintilograma bastante interessante, pois a coluna radioativa mostra, na altura do prolapso, uma diminuição acentuada de sua atividade (Fig. 4, A e B).
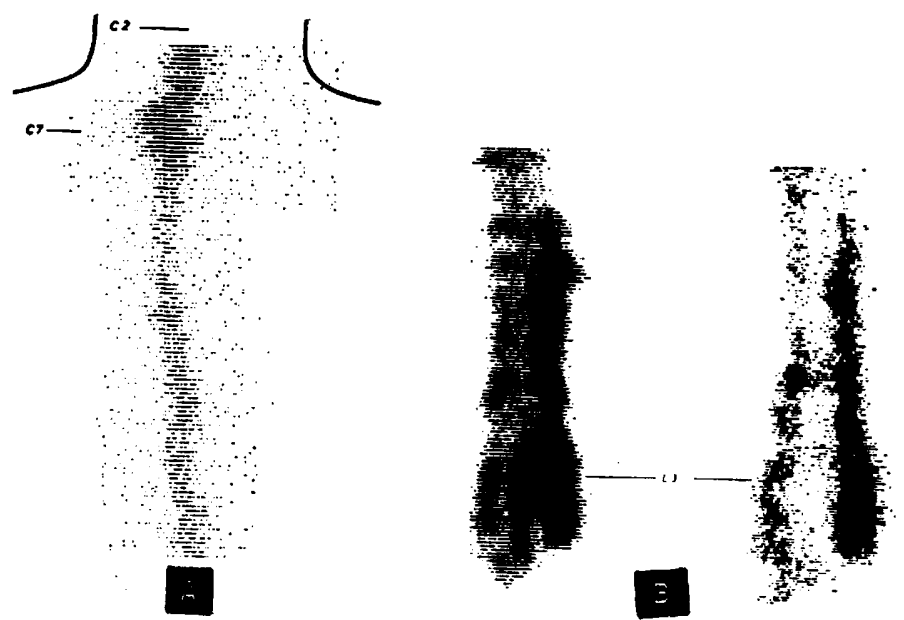

Fig. 5 - Em A, mielocintolograma obtido em paciente com avulsão da 7.a raiz cervical esquerda, correspondendo a dilatação da coluna radioativa observada nesta altura ao enchimento do fundo de saco radicular com a substância radioativa. $E m B$, extravasamento da substância radioativa do espaço subaracnóideo para o peridural através do orifício produzido pela agulha de punção (lombar).

A mielocintilografia demonstrou a existência de prolapso em $30 \%$ dos 127 casos de hérnia discal por nós estudados, sendo que o diagnóstico topográfico exato só pôde ser feito em 20\%. Para ter melhor idéia das possibilidades oferecidas pela mielocintilografia com RIHSA, injetamos, na mes- 
ma sessão, em 67 doentes com hérnias de núcleo pulposo, o iodo radioativo e um meio de contraste positivo, de maneira que os resultados obtidos pelos dois métodos diagnósticos pudessem ser comparados. Os meios de contraste positivos usados foram Abrodil (dois casos), Pantopaque (três) e, nos restantes, um produto ainda em fase experimental [SH 617 (L), da Schering]. Pequenos detalhes foram, como era de esperar, muito mais fàcilmente demonstráveis mediante mielcgrafia com os contrastes positivos. Entretanto, pôde-se verificar, mielocintilogràficamente, hérnias de núcleo pulposo em casos nos quais a mielografia com meios de contraste positivo se apresentava normal.

Outro detalhe que pode ser registrado pela mielocintilografia é o extravasamento de líquido cefalorraqueano após punções lombares ou suboccipitais. Não é raro serem observados extravasamentos da substância radioativa do espaço subaracnóideo para o peridural, sob forma de pequenas coleções de substância radioativa ao lado da coluna radioativa principal, podendo simular dilatação dos fundos de saco radiculares ou pequenas dilataç̃̃es saculares das meninges. O extravasamento pode ser tão intenso a ponto de se observar deposição de quase tôda substância radioativa no espaço peridural (Fig. 5B).

\section{RESUMO}

O estudo de 214 mielocintilografias feitas em 200 pacientes permitiu aos autores chegar às seguintes conclusões: 1) a mielocintilografia com RIHSA - 131I oferece novas possibilidades no campo do diagnóstico neurológico, principalmente no que tange aos processos expansivos, inflamatórios e malformações, sendo limitadas suas possibilidades no diagnóstico de hérnias dos núcleos pulposos; 2) a mielocintilografia não substitui métodos mielográficos convencionais, os dois métodos devendo ser usados de maneira a se completarem; 3) há casos em que a mielocintilografia com RIHSA apresenta vantagem sôbre a mielografia com os meios de contraste positivos, principalmente no diagnóstico de aracnoidites, avulsões de raízes e malformações do canal vertebral; 4) a mielocintilografia com RIHSA tornou-se um método de uso corrente no diagnóstico neurológico, devido à facilidade de execução do exame, à boa tolerância desta substância pelo organismo, à sua rápida absorção e completa eliminação.

\section{SUMMARY}

Myeloscintilography with RIHSA. Value in the diagnosis of pathological processes of the vertebral canal.

The study of 214 myeloscintilographies made on 200 patients have led the authors to the following conclusions: 1) myeloscintilography with RIHSA opens up a series of new possibilities in the field of neurological diagnosis, especially concerning with malformations, inflammatory and expansive processes, its possibilities in cases of disc herniations being of a 
narrower scope; 2) the method does not intend to substitute the conventional myelography with contrast media, both methods having to be used so as to complement each other; 3) there are cases in which myeioscintilography with RIHSA presents advantages over myelography with the positive means of contrast, especially in the diagnosis of arachnitis, nerve avulsions and malformations of the vertebral canal; 4) myeloscintilography with RIHSA has become a method of current use in neurological diagnosis because of the facility with which the examination is made, by the good tolerance of the drug, by its rapid absorption and complete elimination.

\section{ZUSAMMENFASSUNG}

\section{Myeloszintigraphie mit RIHSA in der Diagnostik intraspinaler Erkrankungen.}

Das Studium von 214 Myeloszintigraphien, die in 200 Patienten durchgeführt wurden, lässt feststellen: 1) die Myeloszintigraphie bietet eine Reihe neuer Möglichkeiten in Neurologie, besonders was die Diagnostik raumbeschränkender und arachnoiditischer Prozessen sowie der Missbildungen betrifft; 2) sie kann und will kein Ersatz der Kontrastmittelmyelographie sein, die beiden Methoden Zusammen erweitern das diagnostiche Rüstzeug der Neurologie; 3) in mancher Hinsicht dürfte sie gegenüber der Anwendung von Kontrastmitteln, besonders in der Diagnose von Arachoiditiden, Wurzelausrisse und Missbildungen des Spinalkanals Vorteile haben; 4) sie ist durch technisch leichte Handhabung, gute Verträglichkeit, rasche Resorption und vollständige Elimination der radioaktiven Substanz, und gute diagnostische Leistungsfähigkeit ein beachtenswertes Verfahren.

\section{REFERENCIAS}

1. Bagbio, G. F. \& MORGando, E. - Riv. Pat. Nerv. Ment. 86:7, 1965.

2. BAUER, F. K. \& YUHL, F. T. - Neurology (Minneapolis) 3:341, 1953.

3. BAUER, F. K. \& YUHL, F. T. - Int. J. Appl. Rad. 2:52, 1957.

4. BELL, R. L. \& HERTSCH, G. H. - Int. J. Appl. Rad. 7:19, 1959.

5. DIETZ, H.; WOLF, R. \& ZEITLER, E. - Acta neurochir. (Viena) 13:575, 1965.

6. DIETZ, H.; WOLF, R. \& ZEITLER, E. - Excerpta med. (Amsterdam), Int. Congr. Series 93:236, 1965.

7. DIETZ, H.; ZEITLER, E. \& WOLF, R. - Fortschr. Röntgentr. 105:537, 1966.

8. DIETZ, H.; ZEITLER, E. \& WOLF, R. - Neurochirurgia 9:205, 1966.

9. FRÄNKEL, V. Kh. \& SOSONKIN, Z. S. - Vop. Neirokhir. (Moscou) 28:16, 1964.

10. Gabelowa, N. A.; ARakelow, O. G.; Martusow, E. T. et al. - Med. Radiol. (Moscou) 5:61, 1960 
11. GRos, Ch. M.; WACKENHEIM, A.; VRousos, C. \& SUbIRANA, M. - VII Symposium Neuroradiologicum, New York, setembro 1964.

12. HUBNER, M. D. \& BROWN, D. W. - J. Nucl. Med. 6:465, 1965.

13. KOROLJUK, I. P. - Med. Radiol. (Moscou) 7:40, 1962.

14. KRUPIN, E. N. - Vop. Neirokhir. (Moscou) 20:18, 1955.

15. LIASS, F. M. - Vop Neirokhir. (Moscou) 22:26, 1958.

16. LIASS, F. M. - Radiobiol. Radiother. (Berlin) 4:219, 1963.

17. LIASS, F. M. \& SMAGIN, B. I. - Med. Radiol. (Moscou) 5:51, 1960.

i8. PANTAZIS, G. et al. - II International Symposium of Isotopes, Atenas, 1964.

19. PECKER, J. \& JAVALET, A. - Neuro-Chirurgie (Paris) 6:284, 1960.

20. PERRYMAN, Ch. R.; NOBLE, P. R. \& BRADGON, F. H. - Amer. J. Roentgenol. 80:104, 1958

21. PINTO, F. - Zbl. Neurol. Psychiatr. 171:254, 1963.

22. WACKenheim, A. - J. Méd. de Besançon 1:211, 1955.

23. WACKENHEIM, A. - Radiologe 5:484, 1965.

24. ZEITLER, E; DIETZ, H.; SCHƯRMANN, K. \& WOLF, R. - VII Simpósio Internacional de Bad Gastheim, janeiro 1966. In Radioaktive Isotope in Klinik u. Forschung. Urban \& Schwarzenberg Verlag, Berlin, München, 1966.

\section{Endereços:}

Priv. Doz. Dr. H. Dietz: Neurochirurgische Universitätsklinik - 65, Mainz Deutschland.

Dr. V. Seixas: Rua Coimbra 636 - São Paulo, SP - Brasil. 